\title{
Performance of Garden Pea (Pisum sativum var hortense L.) Genotypes and their Genetic Variation, Genetic Advance, Character Association and Path Analysis for Pod, Seed and Quality Characters
}

\author{
Umesh Thapa $^{1}$, Suman Nandi ${ }^{1 *}$ and Deepsil Gurung ${ }^{2}$ \\ ${ }^{1}$ Department of Vegetable Science, Faculty of Horticulture \\ Bidhan Chandra Krishi Viswavidyalaya, Mohanpur, Nadia -741252, \\ (West Bengal) India \\ ${ }^{2}$ Mirik Bazar, P.o-Mirik, Darjeeling, 734214 \\ *Corresponding author
}

\begin{abstract}
A B S T R A C T
In order to best exploit the available genetic wealth in the crop, the information would have paramount important. Therefore, the present investigation was undertaken to study the best performing variety, nature and magnitude of genetic variability inherently present in the genotypes, and also to ascertain the degree and direction of relationship between yield and its component characters and the direct and indirect effects of various component characters on the yield of twelve genotypes of pea. High GCV and PCV were recorded for total chlorophyll content followed by green pod yield and number of leaves per plant. Heritability was high $(>60 \%)$ for all the characters except pod set per inflorescence, shelling percentage and protein content which exhibited medium heritability. The pod yield was significantly and positively correlated with pod weight, pod girth, pericarp thickness, seed weight per pod, number of pods per plant and weight of 100 seeds. The path coefficient analysis using the phenotypic correlation coefficient among the pair of characters showed that number of pods per plant had the highest positive direct effect on yield. At last it could be concluded that PSM-3 and PSM5 are best performing varieties for this region according to green pod yield.
\end{abstract}

Keywords

Garden pea, Yield, Genetic variation,

Genetic advance,

Character

association and Path analysis

Article Info

Accepted:

26 August 2020

Available Online:

10x September

\section{Introduction}

Garden pea (Pisum sativum var. hortense L.) which belongs to the family Leguminosae, is one of the most popular vegetable crops grown all over the world, both for fresh market and the food processing industry. In
India, it is grown on an area of 530,000 hectares with a total production of 5,345,000 MT (NHB, 2016-2017). West Bengal is the sixth largest garden pea producing state and accounts for $3.5 \%$ of the total production of peas in the country. It is a self-pollinating, diploid crop with chromosome no. $2 \mathrm{n}=14$ 
and with a genome size of $4.4 \mathrm{Gbp}$ (Zhu et al., 2005; Kaur et al., 2012). Pea is very rich in protein $(7.2 \mathrm{~g})$, vitamin A (139 IU), Vitamin C (9 mg), calcium $(20 \mathrm{mg})$, phosphorous (139 mg), energy (81 kcal), carbohydrates $(14.5 \mathrm{~g})$ and sugars $(5.67 \mathrm{~g})$ per $100 \mathrm{~g}$ of edible portion. It is considered as an important cultivated legume next to soyabean, groundnut and beans (Khichi et al., 2016). Besides, it is also rich in lysine, a limiting essential amino acid in cereals. Being a cool season crop, garden pea is extensively grown in cooler climates (Sood et al., 2006).

Green pod yield is the most important economic character as far as garden pea is concerned. There is considerable scope for its expansion but the productivity of pea is still low due to lack of high yielding and multiple disease resistant cultivars. The improvement and production of garden pea crop is needed for the production of quality seeds for this particular region. Scarcity of quality seed is one of the major hindrances faced by the garden pea growers in the plains of West Bengal.

The possibility of improvement in any crop is measured by variability available in the crop (Kumar et al., 2015). Genetic variability is important in plant breeding for improvement of crop plants. Greater the variability in the material more is the genetic potential and greater are the chances of producing a desired type. Hence, it is important, partitioning overall variability into its heritable and nonheritable components with the help of genetic parameters like genetic coefficient of variation, heritability and genetic advance. Knowledge of correlation among different traits and further partitioning them into direct and indirect effects is one of approaches to understand nature and extent of such relationship (Nawab et al., 2008). Keeping the above points in view, there is a constant need to evaluate the germplasm to identify the genetically diverse lines with desirable characters and hence, the present investigation was undertaken.

\section{Materials and Methods}

The present investigation was carried out in New Alluvial Zone of West Bengal, at the open field, in the area of ELP at Bidhan Chandra Krishi Viswavidyalaya, Mohanpur, Nadia (West Bengal) during the year 2016 2017. The location of the experimental site is $23.5^{\circ} \mathrm{N}$ latitude and $80^{\circ} \mathrm{E}$ longitude with an average altitude of $9.75 \mathrm{~m}$ from the mean sea level (MSL). The average temperature ranges from $25-36.5^{0} \mathrm{C}$ during the summer months and $12-25^{\circ} \mathrm{C}$ during the winter months. The average annual rainfall is about $1500 \mathrm{~mm}$. The soil of the experimental plot was sandy loam and slightly acidic in nature with a $\mathrm{pH}$ range of 6.64 to 7.2 .

The 12 genotypes (collected from different sources as represented in the table 1) of garden pea were evaluated for green pod yield during autumn-winter season (2016-2017) in Randomized Block Design with three replications in field condition and spacing was $30 \mathrm{~cm} \times 20 \mathrm{~cm}$. The pre-soaked seeds were sown by dibbling one to two seeds with the depth of $4 \mathrm{~cm}$. The recommended dosage of $\mathrm{N}$, $\mathrm{P}$ and $\mathrm{K}$ (40: 60: $50 \mathrm{~kg}$ per ha) was applied in the form of urea, single super phosphate and murate of potash respectively. A total of five irrigations were given during the total cropping season. The pods were harvested as and when they attained physiological maturity which is indicated by the pod turning yellow in colour. Finally the seeds were cleaned and dried till the moisture content reduced to nine percent.

Different characters of the crop were taken before and after harvesting the crop. Observations were recorded on ten randomly selected equally competitive plants of each 
genotype in each replication. Growth parameters like Plant height $(\mathrm{cm})$, Primary branches per plant, Leaves per plant, Leaf area $\left(\mathrm{cm}^{2}\right)$, Days to first flowering, Days to $50 \%$ flowering, Flowers per inflorescence, Pod set per inflorescence; Pod parameters like Pod length $(\mathrm{cm})$, Pod weight $(\mathrm{g})$, Pod girth (cm), Pericarp thickness $(\mathrm{mm})$, Fresh seed weight per pod (g), Number of pods per plant, Green pod yield per plant (g), Shelling percentage (\%);Seed parameters like Seeds per pod at full maturity, 100 seed weight at full maturity $(\mathrm{g})$ and in pod quality characters like Total sugar content (\%) (Estimated by anthrone method as per Dubois et al., 1951), Reducing sugar (\%) (It was estimated by anthrone method as per Dubois et al., 1951), Total soluble solid (TSS) content of the seeds (A digital refractometer was used for this purpose), Chlorophyll content of immature pods (Chlorophyll a, b and total chlorophyll was estimated as per Sadasivam and Manickam, 1996), Seed protein (The mature dried seeds were used for the estimation of protein content as per Lowry et al., 1951).

All the analysis was done in computer using appropriate programs. Phenotypic variances, genotypic variances and error variances were estimated following Johnson et al., Genotypic and phenotypic coefficients of variation were estimated according to Burton. Broad sense heritability, Genetic advance (GA) and Genetic advance in percent of mean (GAPM) were estimated using the formula suggested by Johnson et al., and Genotypic and phenotypic correlation coefficient were carried out using formula suggested by Miller et al., Johnson et al., and Hanson et al.,

The correlation coefficient was further partitioned into components of direct and indirect effects by path coefficient analysis developed by Wright and later described by Dewey and Lu.

\section{Results and Discussion}

\section{Analysis of variance}

Mean sum of squares presented in Table 2 (a) clearly suggested significant difference of the genotypes for all the characters under study (Gupta et al., 2006, Nawab et al., 2008 and Kumar et al., 2010) which clearly endorsed the justification of studying genetic variability employing these genotypes and characters. Mean coefficient of variation for the 18 characters was low for most of the characters and moderate for only one character viz., pod set per inflorescence (10.33\%)

\section{Mean performance of genotypes}

Mean performances of the 12 genotypes for all the characters presented in the Table 3, 4 and 5 varies significantly. The mean values of various genotypes has also showed wide range of variability for various characters, which were studied in present investigation. The Mean performance of all the characters plant height $(72.13 \mathrm{~cm}, 70.73 \mathrm{~cm})$, number of primary branches per plant $(3.68,3.38)$, number of leaves per plant $(80.09,66.57)$, leaf area $\left(17.80 \mathrm{~cm}^{2}, 15.73 \mathrm{~cm}^{2}\right)$ and main yield attributing characters like pod yield per plant (292.99g, 274.05), seed yield per plant $(154.34 \mathrm{~g}, 146.75 \mathrm{~g}), 100$ seed weight $(24.52 \mathrm{~g}$, $23.35 \mathrm{~g})$, seeds per pod(8.0, 7.80), no of pods per plant $(30.26,32.29)$ were highest for the variety PSM-3 followed by PSM-5. Quality characters like total sugar, reducing sugar and total soluble solid were highest for the variety BK-10 and total protein (\%) was highest in PSM-3.

The total yield was associated with its yield attributes like number of pods per plant (Khichi et al., 2016). Yield is determined by many factors such as soil, climate and agronomic conditions. Crop with vigorous vegetative growth produces higher yield as it 
has higher number of leaves which means more photosynthesis and ultimately results in more yield (Amjad and Anjum, 2002).

\section{Genotypic coefficient of variation}

Studies on the variability using genetic parameters like genotypic coefficient of variation, heritability and genetic advance is essential for initiating an efficient selection programme for any crop. Phenotypic coefficient of variation (PCV) were higher in magnitude than the corresponding genotypic coefficient of variation (GCV) for all the characters studied in present investigation which indicated, noticeable variability in the used genetic material which was not only due to genotypic effect but also due to the effect of environment. Hence, care has to be taken in making selection for these traits on the basis of phenotypic performance alone.

In the present study, low GCV and PCV were observed for plant height $(8.52 \%, 10.60 \%)$, days to first flowering $(9.52 \%, 10.17 \%)$, days to $50 \%$ flowering $(6.29 \% 7.59 \%)$, pod length $(9.07 \%, 9.31 \%)$, shelling percentage $(5.72 \%$, $8.35 \%)$, total sugar content $(8.24 \%, 8.69 \%)$, reducing sugar content $(7.85 \%, 8.33 \%)$, TSS $(7.94 \%, 8.25 \%)$ and dried seed protein $(1.80 \%, 2.51 \%)$ and moderate for characters like number of primary branches $(15.93 \%$, $17.56 \%$ ), pod set per inflorescence $(12.56 \%$, $16.27 \%)$, pod weight $(19.72 \%, 20.61 \%)$, pod girth $(10.10 \%, 11.25 \%)$, pericarp thickness $(16.94 \%, 17.74 \%)$, number of pods per plant $(18.36 \%, 18.96 \%)$, number of seeds per pod at full maturity $(11.79 \%, 13.39 \%), 100$ seed weight (15.42\%, 15.58\%), (Table 5) indicating comparatively less genetic variation among the genotypes for the concerned characters. GCV and PCV estimates were high for number of leaves per plant $(27.27 \%, 27.55 \%)$, leaf area $(22.61 \%$, $23.73 \%$ ), flowers per inflorescence $(22.08 \%$, $24.02 \%)$, seed weight per pod $(23.50 \%$,
$24.13 \%)$, green pod yield per plant $(27.81 \%$, $28.67 \%$ ) and total chlorophyll content $(28.32 \%$, 28.47\%) which suggested comparatively high genetic variation among the genotypes for these characters. High GCV and PCV was recorded for total chlorophyll content followed by green pod yield and number of leaves per plant.

The present findings clearly suggested the worth of these characters for the study of genetic variability in Garden pea. Similar high GCV have been recorded by Nawab et al., (2008), Sharma et al., (2009), Choudhary et al., (2010) and Pal et al., (2013) for green pod yield per plant.

\section{Heritability in broad sense}

Burton (1952) suggested the genetic variability along with heritability should be considered for assessing the maximum and accurate effect of selection. Close correspondence between GCV and PCV estimates was reflected on the broad sense of heritability estimates that were high $(>60 \%)$ for all the characters except pod set per inflorescence $(60.00 \%)$, shelling percentage $(46.88 \%)$ and protein content (52.00) which exhibited medium heritability (31 to $60 \%$ ).

The overall scenario of broad sense heritability estimates for different characters indicated higher efficiency of selection for number of leaves per plant $(97.98 \%)$, leaf area $(90.73 \%)$, pod length (94.99\%), pod weight $(91.25 \%)$, pod girth (95.65\%), pericarp thickness $(90.00 \%)$, seed weight per pod $(91.25 \%)$, number of pods per plant (93.74\%), 100 seed weight at full maturity (97.99\%), green pod yield per plant (94.04), total sugar content $(94.12 \%)$, TSS $(92.82 \%)$ and total chlorophyll content (99.17 \%) (Table 5). However, this broad sense of heritability values are likely to be overestimated as in this calculation it was not 
possible to exclude variation due to different genetic component and their inter-relation.

\section{Genetic advance (GA)}

Johnson et. al. (1955) suggested that heritability in combination with genetic advance would be more reliable in predicting the effect of selection because, genetic advance depends on the amount of genetic variability, the magnitude of masking effect of genetic expression (environmental influence) and the intensity of selection. In the present investigation, genetic advance as percentage of mean indicated few significant traits. Mean genetic advance was high $(>20$ $\%$ ) for number of primary branches per plant (29.76\%), Number of leaves per plant (55.61 $\%)$, leaf area (44.37\%), Flowers per inflorescence (44.52\%), pod weight (38.86 $\%)$, pod girth $(22.15 \%)$, pericarp thickness (33.32\%), seed weight per pod (47.13\%), number of pods per plant (36.61\%), number of seeds per pod at full maturity $(21.37 \%)$, 100 seed weight at full maturity (31.45\%), green pod yield per plant $(55.55 \%)$ and total chlorophyll content $(58.05 \%)$, moderate (10$20 \%$ ) for plant height $(14.12 \%)$, days to first flowering (18.35\%), days to $50 \%$ flowering (10.74\%), pod set per inflorescence (19.99 $\%)$, pod length (18.22 \%), total sugar (16.10 $\%)$, reducing sugar (15.26 \%) and TSS (15.75 $\%)$, and low (<10\%) for shelling percentage $(8.06 \%)$ and protein $(2.67 \%)$ (Table 5). The highest value of genetic advance as mean percent was obtained for number of leaves per plant $(55.61 \%)$ followed by green pod yield per plant $(55.55 \%)$. Similar results of high genetic advance as per cent of mean for green pod yield per plant has been found by Nawab et al., 2008.

\section{Correlations}

The intensity and direction of association among the characters can conveniently be measured by genotypic and phenotypic correlation coefficients. In fact, knowledge of genotypic inter-relationship between characters is also of theoretical interest genotypic correlation may be derived from genetic linkage, pleiotropy or developmentally induced relationship between components which are only indirectly the consequence of gene action (Adams, 1967).

The genotypic and phenotypic correlation coefficients among the pair of characters have been presented in Table 6 and 7 respectively. However, phenotypic correlation coefficients between the pair of characters have been utilized to study the character association ship. In the present investigation, pod yield was significantly and positively correlated with pod weight $(0.801)$, pod girth (0.777), pericarp thickness (0.578), seed weight per pod (0.758), number of pods per plant (0.784) and weight of 100 seeds (0.595).

A number of earlier studies identified these characters along with some others showing strong positive correlation with pod yield (Kumar et al., 2006 and Kumar et al., 2010). Therefore, pod weight, pod girth, pericarp thickness, seed weight per pod, number of pods per plant and weight of 100 seeds emerged as important characters to be considered for indirect selection in the yield improvement programme.

However, it was also observed that pod yield was significantly and negatively correlated with leaf area (-0.565), flowers per inflorescence $(-0.541)$ and pod set per inflorescence (-0.538) (Table 7). A number of earlier studies identified these characters along with some others showing strong positive correlation with pod yield (Kumar et al., 2006, Kumar et al., 2010 and Singh et al., 2018). 


\section{Path co-efficient}

Linear correlation between any two characters may present a confusing picture regarding character association ship because any character may have simultaneous influence on many characters of the plant. Path co-efficient technique is more useful in establishing direct and indirect relationship among many characters which present a more realistic interpretation of the characters influencing a particular trait.

The path coefficient analysis using phenotypic correlation coefficient among pair of characters depicting direct and indirect effect on pod yield has been presented in the Table 8 . The results of path analysis revealed that number of pods per plant had the highest positive direct effect $(0.597)$ on yield followed by pod weight $(0.320)$ and seed weight per pod (0.164).

Mean residual effect was 0.01 suggesting the inclusion of $99.99 \%$ pod yield components in this study, which suggested the high validity of such study employing these characters. Similar results were obtained by Nawab et al., (2008), Sharma et al., (2009) Kumar et al., (2010) and Karnwal et al., (2013).

Table.1 Sources of different Garden pea genotypes used as experimental Materials

\begin{tabular}{|r|c|c|}
\hline Sl. No. & Genotypes & Sources \\
\hline $\mathbf{0 1 .}$ & Arkel & I.A.R.I., New Delhi \\
\hline $\mathbf{0 2}$. & Pusa Shree & I.A.R.I., New Delhi \\
\hline $\mathbf{0 3 .}$ & Arka Pramod & I.I.H.R., Bangalore \\
\hline $\mathbf{0 4}$. & Arka Kartik & I.I.H.R., Bangalore \\
\hline $\mathbf{0 5}$. & Arka Apoorva & I.I.H.R., Bangalore \\
\hline $\mathbf{0 6}$ & Arka Ajit & I.I.H.R., Bangalore \\
\hline $\mathbf{0 7}$ & Pant Uphar & GBPUAT, Pantnagar \\
\hline $\mathbf{0 8 .}$ & PSM-5 & GBPUAT, Pantnagar \\
\hline $\mathbf{0 9 .}$ & Azad Pea- 3 & GBPUAT, Pantnagar \\
\hline $\mathbf{1 0 .}$ & PSM-3 & GBPUAT, Pantnagar \\
\hline $\mathbf{1 1 .}$ & BK-10 & GBPUAT, Pantnagar \\
\hline $\mathbf{1 2}$. & Mohanpur Local & Nadia, West Bengal \\
\hline
\end{tabular}


Table.2(a) Analysis of variance for pod and yield components in 12 genotypes of Garden pea

\begin{tabular}{|c|c|c|c|c|c|c|c|c|c|c|c|c|c|c|c|c|c|c|}
\hline \multirow[b]{2}{*}{$\begin{array}{l}\text { Sources } \\
\text { of } \\
\text { Variation } \\
\text { (d.f.) }\end{array}$} & \multicolumn{18}{|c|}{ Mean Sum of Square (MSS) } \\
\hline & 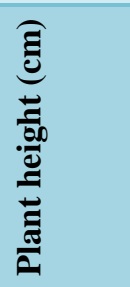 & 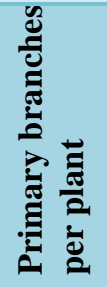 & 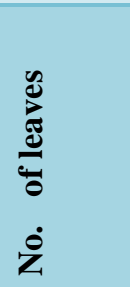 & ָే & 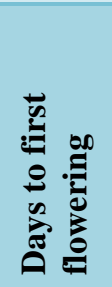 & 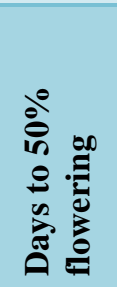 & 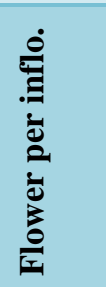 & 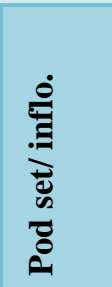 & 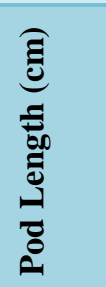 & 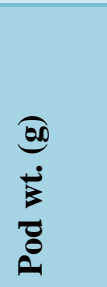 & 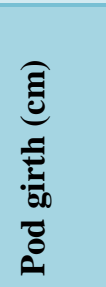 & 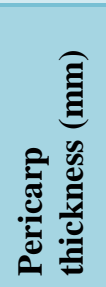 & 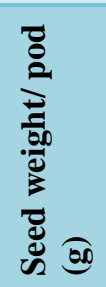 & $\begin{array}{l}\vec{E} \\
\frac{\pi}{2} \\
\frac{2}{0} \\
0\end{array}$ & 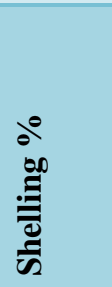 & 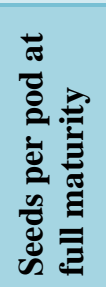 & 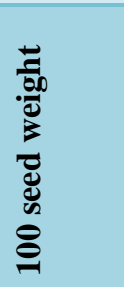 & 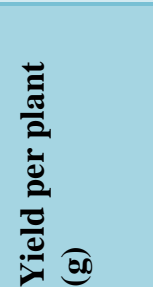 \\
\hline $\begin{array}{l}\text { Replication } \\
\text { (2) }\end{array}$ & 25.810 & 0.036 & 1.7444 & 0.827 & 8.180 & 6.730 & 0.043 & 0.010 & 0.019 & 0.139 & 0.002 & 0.005 & 0.008 & 1.029 & 5.228 & 0.035 & 0.080 & 26.647 \\
\hline $\begin{array}{c}\text { Genotypes } \\
\text { (11) }\end{array}$ & $\begin{array}{l}106.106 \\
*\end{array}$ & $\begin{array}{l}\mathbf{0 . 6 5 0} \\
* *\end{array}$ & $\begin{array}{l}\text { 578.49* } \\
*\end{array}$ & $\begin{array}{l}24.6 \\
17 * *\end{array}$ & $\begin{array}{l}72.12 \\
2 * *\end{array}$ & $\begin{array}{l}51.18 \\
7 * *\end{array}$ & 0.441 & $\begin{array}{l}\mathbf{0 . 1 0 6} \\
*\end{array}$ & $\begin{array}{l}2.090 \\
* *\end{array}$ & $\begin{array}{l}7.433 \\
* *\end{array}$ & $\begin{array}{l}\text { 0.664 } \\
* *\end{array}$ & $\begin{array}{l}0.274 \\
* *\end{array}$ & $\begin{array}{l}2.319 \\
* *\end{array}$ & $\begin{array}{l}\text { 65.341* } \\
*\end{array}$ & 25.227 & $\begin{array}{l}2.399 \\
* *\end{array}$ & $\begin{array}{l}28.467 * \\
*\end{array}$ & $\begin{array}{l}\text { 8934.012* } \\
*\end{array}$ \\
\hline Error (22) & 16.327 & 0.044 & 3.942 & 0.807 & 3.261 & 6.749 & 0.016 & 0.020 & 0.036 & 0.223 & 0.010 & 0.009 & 0.041 & 1.423 & 8.166 & 0.212 & 0.202 & 184.754 \\
\hline CV (\%) & 6.295 & 7.396 & 3.912 & 7.207 & 3.588 & 4.247 & 7.605 & 10.336 & 2.084 & 6.001 & 2.374 & 5.268 & 5.492 & 4.743 & 6.513 & 6.348 & 2.218 & 6.999 \\
\hline
\end{tabular}

Table.2(b) Analysis of variance for pod and seed quality characters in 12 genotypes

$$
\text { of Garden pea }
$$

\begin{tabular}{|c|c|c|c|c|c|}
\hline \multirow{2}{*}{$\begin{array}{c}\text { Sources of } \\
\text { Variation (d.f.) }\end{array}$} & \multicolumn{5}{|c|}{ Mean Sum of Square (MSS) } \\
\hline & $\begin{array}{c}\text { Total } \\
\text { sugar }(\%)\end{array}$ & $\begin{array}{l}\text { Reducing } \\
\text { sugar }(\%)\end{array}$ & $\begin{array}{l}\text { Total soluble } \\
\text { solids ( }{ }^{\circ} \text { Brix) }\end{array}$ & $\begin{array}{c}\text { Total chlorophyll } \\
\text { (mg) }\end{array}$ & $\begin{array}{c}\text { Protein } \\
(\%)\end{array}$ \\
\hline Replication (2) & 0.035 & 0.009 & 0.280 & 0.047 & 0.222 \\
\hline Genotypes (11) & $0.483 * *$ & $0.127 * *$ & $5.971 * *$ & $14.372 * *$ & $0.506^{*}$ \\
\hline Error (22) & 0.017 & 0.005 & 0.153 & 0.048 & 0.121 \\
\hline CV $(\%)$ & 2.759 & 2.767 & 2.234 & 2.851 & 2.797 \\
\hline
\end{tabular}


Table.3 Performance of the Garden pea genotypes for pod and yield components

\begin{tabular}{|c|c|c|c|c|c|c|c|c|c|c|c|c|c|c|c|c|c|c|c|}
\hline Genotypes & $\begin{array}{c}\text { Plant } \\
\text { height } \\
(\mathrm{cm})\end{array}$ & $\begin{array}{c}\text { Primary } \\
\text { branches/ } \\
\text { plant }\end{array}$ & $\begin{array}{l}\text { No. of } \\
\text { leaves }\end{array}$ & $\begin{array}{c}\text { Leaf } \\
\text { Area } \\
\left(\mathrm{cm}^{2}\right)\end{array}$ & $\begin{array}{c}\text { Days to } \\
\text { first } \\
\text { flowering }\end{array}$ & $\begin{array}{c}\text { Days to } \\
50 \% \\
\text { flowering }\end{array}$ & 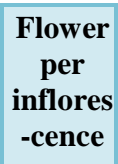 & $\begin{array}{l}\text { Pod set/ } \\
\text { inflores } \\
- \text { cence }\end{array}$ & $\begin{array}{c}\text { Pod } \\
\text { Length } \\
(\mathrm{cm})\end{array}$ & \begin{tabular}{|c|} 
Pod \\
weight \\
(g)
\end{tabular} & $\begin{array}{l}\text { Pod } \\
\text { girth } \\
(\mathrm{cm})\end{array}$ & $\begin{array}{c}\text { Pericarp } \\
\text { thick- } \\
\text { ness } \\
(\mathrm{mm})\end{array}$ & $\begin{array}{c}\text { Seed } \\
\text { weight } / \\
\text { pod } \\
(\text { g) }\end{array}$ & $\begin{array}{l}\text { No. of } \\
\text { pods/ } \\
\text { plant }\end{array}$ & $\begin{array}{c}\text { Shelling } \\
\%\end{array}$ & $\begin{array}{c}\text { Seeds pel } \\
\text { pod at } \\
\text { full } \\
\text { maturity }\end{array}$ & $\begin{array}{c}100 \text { seed } \\
\text { weight at } \\
\text { full } \\
\text { maturity }\end{array}$ & $\begin{array}{c}\text { Seed } \\
\text { yield } \\
\text { per } \\
\text { plant }\end{array}$ & $\begin{array}{c}\text { Pod } \\
\text { yield } \\
\text { per } \\
\text { plant }\end{array}$ \\
\hline Arkel & 51.34 & 3.35 & 30.28 & 12.96 & 47.00 & 59.06 & 2.25 & 1.44 & 8.60 & 6.64 & 4.15 & 2.15 & 2.91 & 29.10 & 43.04 & 6.10 & 18.58 & 84.73 & 193.21 \\
\hline Pusa & 72.13 & 2.55 & 36.70 & 12.05 & 43.60 & 61.05 & 2.22 & 1.16 & 7.16 & 6.59 & 4.16 & 1.54 & 2.64 & 25.93 & 45.51 & 5.50 & 25.47 & 70.02 & 170.85 \\
\hline Arka & 70.73 & 2.81 & 80.09 & 12.79 & 49.65 & 62.10 & 1.45 & 1.16 & 8.85 & 6.81 & 3.85 & 1.23 & 3.69 & 21.05 & 52.46 & 6.95 & 17.55 & 77.91 & 144.06 \\
\hline Arka & 61.62 & 2.52 & 39.51 & 15.73 & 48.20 & 57.82 & 1.53 & 1.48 & 9.75 & 6.11 & 3.60 & 1.49 & 2.65 & 25.77 & 43.73 & 8.70 & 18.13 & 65.63 & 157.18 \\
\hline $\begin{array}{l}\text { Arka } \\
\text { Apoorva }\end{array}$ & 67.57 & 2.92 & 66.57 & 17.80 & 53.22 & 63.59 & 1.58 & 1.40 & 9.40 & 7.67 & 3.85 & 1.80 & 3.96 & 20.19 & 49.86 & 7.40 & 17.40 & 79.87 & 154.97 \\
\hline Arka Ajit & 62.39 & 2.92 & 45.17 & 13.52 & 53.93 & 65.56 & 1.63 & 1.59 & 9.96 & 6.10 & 3.80 & 1.76 & 2.74 & 22.54 & 49.54 & 6.40 & 18.44 & 61.45 & 137.48 \\
\hline Pant & 69.59 & 2.88 & 56.99 & 8.92 & 58.65 & 66.07 & 1.92 & 1.60 & 8.55 & 9.54 & 4.80 & 1.98 & 3.97 & 16.54 & 45.66 & 7.30 & 23.16 & 65.40 & 165.63 \\
\hline PSM-5 & 59.79 & 3.38 & 60.88 & 9.15 & 50.24 & 68.07 & 1.34 & 1.18 & 8.90 & 10.33 & 4.63 & 1.65 & 4.78 & 32.29 & 45.65 & 7.80 & 23.35 & 146.75 & 274.05 \\
\hline Azad Pea- & 66.50 & 2.21 & 53.40 & 9.10 & 58.60 & 59.89 & 1.19 & 1.15 & 9.01 & 8.85 & 4.55 & 1.79 & 4.98 & 29.34 & 50.84 & 7.40 & 21.46 & 146.00 & 259.15 \\
\hline PSM-3 & 67.77 & 2.35 & 51.12 & 9.60 & 49.40 & 59.11 & 1.08 & 1.14 & 9.65 & 10.16 & 5.01 & 2.25 & 4.85 & 30.26 & 48.69 & 8.00 & 24.52 & 154.34 & 292.99 \\
\hline BK-10 & $\mathbf{5 9 . 9 0}$ & 3.68 & 45.04 & 13.00 & 46.23 & 53.38 & 2.04 & 1.59 & 10.40 & 6.88 & 3.85 & 1.66 & 3.20 & 22.84 & 43.68 & 8.00 & 16.57 & 72.68 & 156.03 \\
\hline $\begin{array}{l}\text { Mohanpur } \\
\text { Local }\end{array}$ & 60.87 & 2.30 & 43.16 & 14.93 & 45.19 & 58.38 & 1.60 & 1.32 & 9.20 & 8.69 & 4.70 & 1.88 & 4.14 & 25.90 & 44.93 & 7.40 & 18.38 & 107.20 & 224.94 \\
\hline S.Em \pm & 2.33 & 0.12 & 1.15 & 0.52 & 1.05 & 1.50 & 0.07 & 0.08 & 0.11 & 0.27 & 0.06 & 0.05 & 0.12 & 0.69 & 1.78 & 0.27 & 0.26 & 2.79 & 7.85 \\
\hline CD at $5 \%$ & 6.84 & 0.35 & 3.36 & 1.52 & 3.06 & 4.40 & 0.21 & 0.24 & 0.32 & 0.80 & 0.17 & 0.16 & 0.34 & 2.02 & 5.22 & 0.78 & 0.76 & 8.07 & 23.02 \\
\hline
\end{tabular}


Table.4 Performance of Garden pea genotypes for pod and seed quality characters

\begin{tabular}{|c|c|c|c|c|c|}
\hline Genotypes & $\begin{array}{c}\text { Total } \\
\text { sugar }(\%)\end{array}$ & $\begin{array}{l}\text { Reducing } \\
\text { sugar }(\%)\end{array}$ & $\begin{array}{l}\text { Total soluble } \\
\text { solids ( }{ }^{0} \text { Brix) }\end{array}$ & Total chlorophyll (mg) & Protein $(\%)$ \\
\hline Arkel & 4.15 & 2.06 & 17.33 & 8.35 & 19.86 \\
\hline Pusa Shree & 4.85 & 2.62 & 16.23 & 11.64 & 20.04 \\
\hline Arka Pramod & 5.09 & 2.74 & 20.63 & 6.73 & 19.45 \\
\hline Arka Kartik & 4.77 & 2.53 & 19.16 & 6.09 & 19.69 \\
\hline Arka Apoorva & 4.60 & 2.39 & 18.43 & 6.09 & 20.23 \\
\hline Arka Ajit & 5.25 & 2.63 & 15.90 & 5.60 & 20.34 \\
\hline Pant Uphar & 4.61 & 2.63 & 16.00 & 10.88 & 19.46 \\
\hline PSM 5 & 4.01 & 2.40 & 17.00 & 9.13 & 19.65 \\
\hline Azad Pea 3 & 4.71 & 2.53 & 16.80 & 9.32 & 20.49 \\
\hline PSM 3 & 5.10 & 2.65 & 18.46 & 8.08 & 20.33 \\
\hline BK 10 & 5.30 & 2.76 & 16.93 & 5.31 & 19.43 \\
\hline $\begin{array}{l}\text { Mohanpur } \\
\text { Local }\end{array}$ & 4.90 & 2.81 & 17.46 & 5.33 & 19.34 \\
\hline S.Em \pm & 0.08 & 0.04 & 0.23 & 0.12 & 0.20 \\
\hline CD at $5 \%$ & 0.22 & 0.12 & 0.66 & 0.37 & 0.59 \\
\hline
\end{tabular}


Table.5 Estimates of Variability, Heritability and Genetic Advance as per cent of mean for 23 characters in 12 genotypes of Garden pea

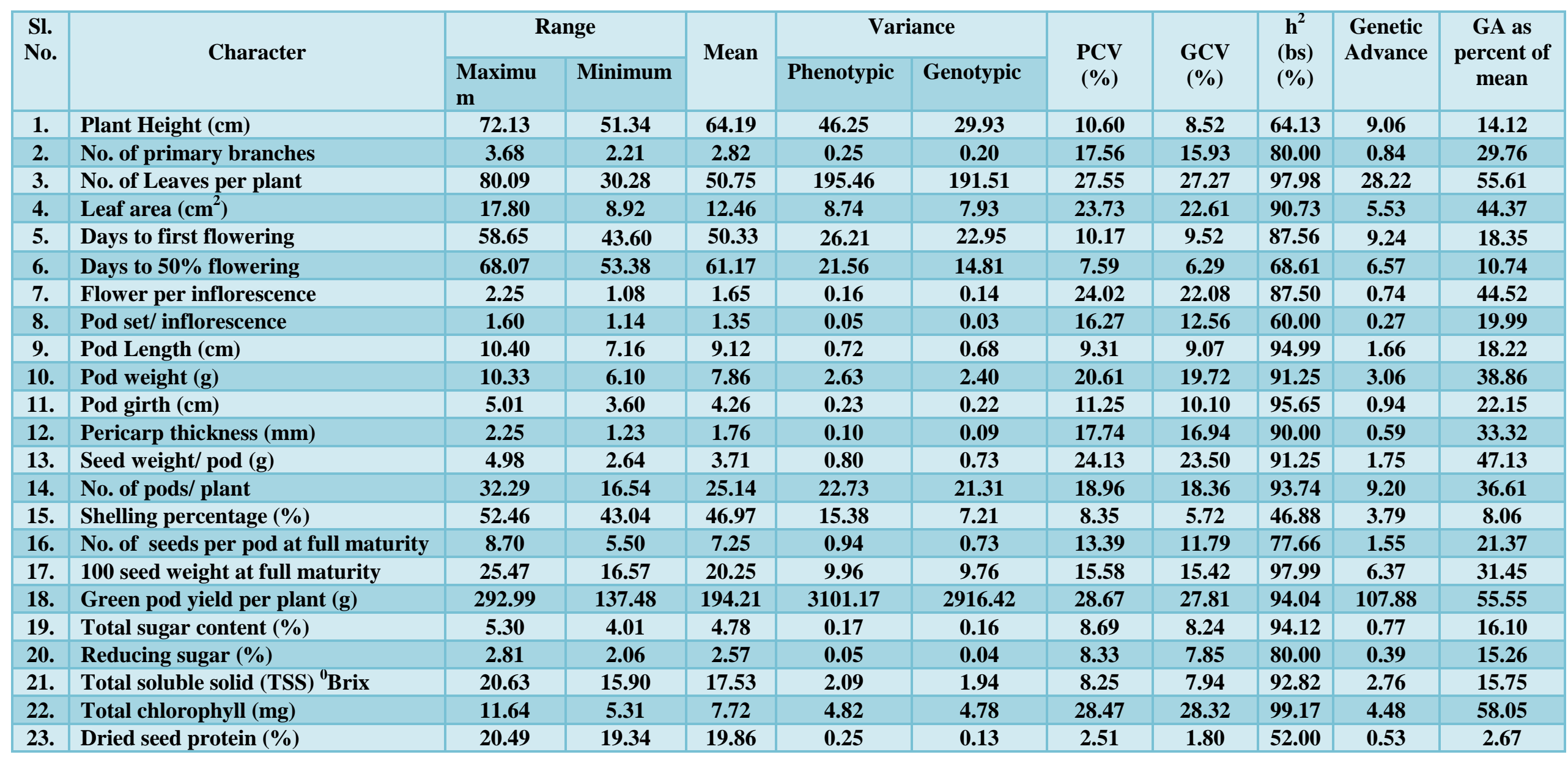


Table.6 Genotypic correlation co-efficient for growth and yield components

\begin{tabular}{|c|c|c|c|c|c|c|c|c|c|c|c|c|}
\hline Character & $\begin{array}{c}\text { Plant } \\
\text { height } \\
\text { (cm) }\end{array}$ & $\begin{array}{l}\text { Leaf } \\
\text { area } \\
\left(\mathrm{cm}^{2}\right)\end{array}$ & $\begin{array}{c}\text { Flowers } \\
\text { per } \\
\text { inflores } \\
\text { ce-nce }\end{array}$ & $\begin{array}{l}\text { Pod set } \\
\text { per } \\
\text { infloresc } \\
\text { e-nce }\end{array}$ & $\begin{array}{c}\text { Pod } \\
\text { length } \\
(\mathrm{cm})\end{array}$ & $\begin{array}{c}\text { Pod } \\
\text { weight } \\
\text { (g) }\end{array}$ & $\begin{array}{l}\text { Pod } \\
\text { girth } \\
(\mathrm{cm})\end{array}$ & $\begin{array}{c}\text { Pericar } \\
\text { p } \\
\text { thickne } \\
\text { ss }(\mathbf{m m})\end{array}$ & $\begin{array}{c}\text { Seed } \\
\text { weight } \\
\text { per pod } \\
\text { (g) }\end{array}$ & $\begin{array}{c}\text { No. of } \\
\text { pods } \\
\text { per } \\
\text { plant }\end{array}$ & $\begin{array}{c}\text { No. of } \\
\text { seeds } \\
\text { per pod }\end{array}$ & $\begin{array}{c}\text { Weight } \\
\text { of } 100 \\
\text { seeds } \\
\text { (g) }\end{array}$ \\
\hline Leaf area $\left(\mathrm{cm}^{2}\right)$ & -0.198 & & & & & & & & & & & \\
\hline $\begin{array}{l}\text { Flowers per } \\
\text { inflorescence }\end{array}$ & -0.270 & 0.215 & & & & & & & & & & \\
\hline $\begin{array}{l}\text { Pod set per } \\
\text { inflorescence }\end{array}$ & -0.414 & 0.343 & 0.486 & & & & & & & & & \\
\hline Pod length (cm) & -0.372 & 0.291 & -0.396 & 0.487 & & & & & & & & \\
\hline Pod weight (g) & 0.168 & ${ }^{-}$ & -0.560 & -0.442 & -0.068 & & & & & & & \\
\hline Pod girth $(\mathrm{cm})$ & 0.139 & $\stackrel{-}{0.712}$ & -0.331 & -0.418 & -0.254 & 0.916 ** & & & & & & \\
\hline $\begin{array}{l}\text { Pericarp thickness } \\
\text { (mm) }\end{array}$ & -0.349 & -0.251 & -0.006 & 0.149 & 0.094 & 0.462 & 0.618 & & & & & \\
\hline Seed weight per pod (g) & 0.218 & -0.538 & $-0.744^{* *}$ & $-0.580^{* *}$ & 0.075 & $0.922^{* *}$ & $0.756^{* *}$ & 0.298 & & & & \\
\hline No. of pods per plant & -0.454 & -0.325 & -0.328 & $-0.637^{*}$ & -0.074 & 0.311 & 0.345 & 0.272 & 0.341 & & & \\
\hline No. of seeds per pod & -0.163 & 0.002 & $-0.600^{*}$ & 0.111 & $0.645^{*}$ & 0.350 & 0.015 & -0.126 & 0.412 & 0.102 & & \\
\hline Weight of 100 seeds (g) & 0.518 & $\stackrel{-}{0.734^{*}}$ & -0.139 & $-0.535^{*}$ & $-0.607^{*}$ & 0.535 & $0.681^{*}$ & 0.313 & 0.370 & 0.323 & -0.280 & \\
\hline $\begin{array}{c}\text { Green pod yield per } \\
\text { plant }(\mathrm{g})\end{array}$ & -0.121 & $0 . \overline{597}$ & $-0.590^{*}$ & $-0.690^{* *}$ & -0.058 & $0.805^{* *}$ & $0.808^{* * *}$ & 0.500 & $0.809^{* *}$ & $0.790^{* *}$ & 0.261 & 0.552 \\
\hline
\end{tabular}

(*Values ranged from $\geq 0.575$ to $\leq 0.707$, significant at $0.05 \%$ level of probability; $* *$ Values $\geq 0.707$, significant at $0.01 \%$ level of probability) 
Table.7 Phenotypic correlations co-efficient for growth and yield components

\begin{tabular}{|c|c|c|c|c|c|c|c|c|c|c|c|c|}
\hline Character & $\begin{array}{c}\text { Plant } \\
\text { height } \\
\text { (cm) }\end{array}$ & $\begin{array}{l}\text { Leaf } \\
\text { area } \\
\left(\mathrm{cm}^{2}\right)\end{array}$ & $\begin{array}{c}\text { Flowers } \\
\text { per } \\
\text { inflores } \\
\text { ce-nce }\end{array}$ & $\begin{array}{c}\text { Pod set } \\
\text { per } \\
\text { infloresc } \\
\text { ence }\end{array}$ & $\begin{array}{c}\text { Pod } \\
\text { length } \\
(\mathbf{c m})\end{array}$ & $\begin{array}{c}\text { Pod } \\
\text { weight } \\
\text { (g) }\end{array}$ & $\begin{array}{l}\text { Pod } \\
\text { girth } \\
(\mathrm{cm})\end{array}$ & $\begin{array}{c}\text { Pericar } \\
\text { p } \\
\text { thickne } \\
\text { ss }(\mathbf{m m})\end{array}$ & $\begin{array}{c}\text { Seed } \\
\text { weight } \\
\text { per pod } \\
\text { (g) }\end{array}$ & $\begin{array}{c}\text { No. of } \\
\text { pods } \\
\text { per } \\
\text { plant }\end{array}$ & $\begin{array}{c}\text { No. of } \\
\text { seeds } \\
\text { per pod }\end{array}$ & $\begin{array}{c}\text { Weight } \\
\text { of } 100 \\
\text { seeds } \\
\text { (g) }\end{array}$ \\
\hline Leaf area $\left(\mathrm{cm}^{2}\right)$ & -0.138 & & & & & & & & & & & \\
\hline $\begin{array}{l}\text { Flowers per } \\
\text { inflorescence }\end{array}$ & -0.199 & 0.203 & & & & & & & & & & \\
\hline $\begin{array}{l}\text { Pod set per } \\
\text { inflorescence }\end{array}$ & -0.292 & 0.322 & 0.500 & & & & & & & & & \\
\hline Pod length (cm) & -0.317 & 0.262 & -0.347 & 0.391 & & & & & & & & \\
\hline Pod weight (g) & 0.134 & $\stackrel{-}{0.594^{*}}$ & -0.477 & -0.301 & -0.031 & & & & & & & \\
\hline Pod girth $(\mathrm{cm})$ & 0.111 & $\stackrel{-}{0.670}^{*}$ & -0.304 & -0.339 & -0.229 & $0.870^{* *}$ & & & & & & \\
\hline $\begin{array}{l}\text { Pericarp thickness } \\
\text { (mm) }\end{array}$ & -0.308 & -0.216 & 0.035 & 0.169 & 0.114 & 0.449 & $0.586^{*}$ & & & & & \\
\hline Seed weight per pod (g) & 0.165 & -0.482 & $-0.692^{*}$ & -0.456 & 0.063 & $0.862^{* *}$ & $0.730^{* * *}$ & 0.308 & & & & \\
\hline No. of pods per plant & -0.361 & -0.333 & -0.325 & -0.521 & -0.062 & 0.303 & 0.335 & 0.213 & 0.304 & & & \\
\hline No. of seeds per pod & -0.087 & 0.046 & -0.467 & 0.090 & $0.597^{*}$ & 0.295 & 0.050 & -0.059 & 0.392 & 0.065 & & \\
\hline Weight of 100 seeds (g) & 0.434 & $\overline{-}^{0.704}$ & -0.130 & -0.461 & $-0.575^{*}$ & 0.508 & $0.659^{*}$ & 0.296 & 0.354 & 0.314 & -0.226 & \\
\hline $\begin{array}{c}\text { Green pod yield per } \\
\text { plant (g) }\end{array}$ & -0.081 & -0.565 & -0.541 & -0.538 & -0.032 & $0.801^{* *}$ & $0.777^{* *}$ & $0.458^{* *}$ & $0.758^{* *}$ & $0.784^{* *}$ & 0.214 & 0.535 \\
\hline
\end{tabular}


Table.8 Path co-efficient analysis of the components of pod yield per plant at phenotypic level

\begin{tabular}{|c|c|c|c|c|c|c|c|c|c|c|c|c|}
\hline Character & $\begin{array}{c}\text { Plant } \\
\text { height } \\
(\mathrm{cm})\end{array}$ & $\begin{array}{l}\text { Leaf } \\
\text { area } \\
\left(\mathrm{cm}^{2}\right)\end{array}$ & $\begin{array}{c}\text { Flowers } \\
\text { per } \\
\text { inflores } \\
\text { cence }\end{array}$ & $\begin{array}{l}\text { Pod set } \\
\text { per } \\
\text { inflore } \\
\text { scence }\end{array}$ & $\begin{array}{c}\text { Pod } \\
\text { length } \\
(\mathrm{cm})\end{array}$ & $\begin{array}{c}\text { Pod } \\
\text { weight } \\
\text { (g) }\end{array}$ & $\begin{array}{l}\text { Pod } \\
\text { girth } \\
(\mathrm{cm})\end{array}$ & $\begin{array}{l}\text { Peri } \\
\text { carp } \\
\text { thick } \\
\text { ness } \\
(\mathrm{mm})\end{array}$ & $\begin{array}{c}\text { Seed } \\
\text { weight } \\
\text { per } \\
\text { pod }(g)\end{array}$ & $\begin{array}{c}\text { No. of } \\
\text { pods } \\
\text { per } \\
\text { plant }\end{array}$ & $\begin{array}{c}\text { No. of } \\
\text { seeds } \\
\text { per } \\
\text { pod }\end{array}$ & $\begin{array}{c}\text { Weig } \\
\text { ht of } \\
100 \\
\text { seeds } \\
\text { (g) }\end{array}$ \\
\hline Leaf area $\left(\mathrm{cm}^{2}\right)$ & 0.071 & -0.007 & -0.004 & 0.008 & -0.037 & 0.043 & 0.018 & -0.014 & 0.027 & -0.216 & 0.002 & 0.026 \\
\hline $\begin{array}{l}\text { Flowers per } \\
\text { inflorescence }\end{array}$ & -0.010 & 0.050 & 0.004 & 09 & 0.030 & -0.190 & -0.108 & -0.010 & -0.079 & -0.199 & 0.001 & -0.043 \\
\hline $\begin{array}{l}\text { Pod set per } \\
\text { inflorescence }\end{array}$ & -0.014 & 0.010 & 0.019 & -0.014 & -0.040 & -0.153 & -0.049 & 0.002 & -0.114 & -0.194 & 0.013 & -0.008 \\
\hline Pod length $(\mathrm{cm})$ & -0.021 & 0.016 & 0.010 & -0.028 & 0.045 & -0.096 & -0.055 & 0.008 & -0.075 & -0.311 & -0.003 & -0.028 \\
\hline Pod weight (g) & -0.023 & 0.013 & -0.007 & -0.011 & 0.115 & -0.010 & -0.037 & 0.005 & 0.010 & -0.037 & -0.017 & -0.035 \\
\hline Pod girth (cm) & 0.010 & -0.030 & -0.009 & 0.008 & -0.004 & 0.320 & 0.140 & 0.021 & 0.141 & 0.181 & -0.008 & 0.031 \\
\hline $\begin{array}{c}\text { Pericarp } \\
\text { thickness }(\mathrm{mm})\end{array}$ & 0.008 & -0.033 & -0.000 & 0.0 & -0.026 & 0.278 & & 0.027 & & 200 & 0.001 & 0.040 \\
\hline $\begin{array}{c}\text { Seed weight per } \\
\operatorname{pod}(\mathrm{g})\end{array}$ & -0.022 & -0.011 & 0.001 & -0.005 & 0.013 & 0.143 & 0.095 & 0.046 & 0.051 & 0.127 & 0.002 & 0.018 \\
\hline $\begin{array}{c}\text { No. of pods per } \\
\text { plant }\end{array}$ & 0.012 & -0.024 & -0.013 & 0.013 & 0.007 & 0.275 & 0.118 & 0.014 & 0.164 & 0.182 & -0.011 & 0.022 \\
\hline $\begin{array}{c}\text { No. of seeds per } \\
\text { pod }\end{array}$ & -0.026 & -0.017 & -0.006 & 0.015 & -0.007 & 0.097 & 0.054 & 0.010 & 0.050 & 0.597 & -0.002 & 0.019 \\
\hline $\begin{array}{l}\text { Weight of } 100 \\
\text { seeds (g) }\end{array}$ & -0.006 & 0.002 & -0.009 & -0.003 & 0.069 & 0.094 & 0.008 & -0.003 & 0.064 & 0.039 & -0.028 & -0.014 \\
\hline $\begin{array}{c}\text { Green pod yield } \\
\text { per plant (g) }\end{array}$ & 0.031 & -0.035 & -0.003 & 0.013 & -0.066 & 0.162 & 0.106 & 0.014 & 0.058 & 0.188 & 0.006 & 0.061 \\
\hline
\end{tabular}


In conclusion, all the morphological traits showed highly significant variations among the genotypes and the variations could be used in plant improvement program. High GCV and PCV was recorded for total chlorophyll content followed by green pod yield and number of leaves per plant and selection for the improvement of these traits could be effective. Furthermore, among studied traits, six traits viz, pod weight, pod girth, pericarp thickness, seed weight per pod, number of pods per plant and weight of 100 seeds showed highly positive correlation and positive direct effect on yield. Number of pods per plant had the highest positive direct effect on yield followed by pod weight and seed weight per pod. Therefore, emphasis should be given on these traits during selection in breeding program in order to increase green pod yield. In respective to green pod yield and all other characteristics, it could be concluded that variety PSM-3 and PSM-5 are best for this particular region.

\section{References}

Adams, M.W. (1967). Basis of yield component compensation in crop plants with special reference to field bean (Phaseolus vulgaris). Crop Science, 7: 505-510.

Amjad, M. and Anjum, M.A. (2002). Performance of nine pea cultivars under Faisalabad conditions. Pakistan journal of Agricultural Sciences, 39: 6-19.

Burton, G.W. (1952). Quantitative inheritance in grasses. In: Proc. Sixth Int. Grassland Congress. pp. 277-283.

Choudhary, H., Verma, M.K. and Sofi, A.A. (2010). Genetic variability, heritability and genetic advance for yield components in garden pea. Pantnagar Journal of Research, 8(2):195-197.

Dewey, J.R. and Lu, K.H. (1959). Correlation and path coefficient analysis of components of crested wheat grass seed production. Agronomy Journal, 51: 515-518.

Gupta, A.J., Singh, Y.V. and Verma, T.S. (2006). Genetic variability and heritability in garden pea (Pisum sativum L.). Indian Journal of Horticulture, 63(3) 332-334.

Johnson, H.W., Robinson, H.F. and Comstock, R.E. (1955). Estimates of genetic and environmental variability of soybean. Agronomy Journal, 47: 314-318.

Karnwal, M.K., Rai, R., Singh, D., Singh, V.P., Pal, M. and Kumar, A. (2013). Genetic variability in garden pea under rainfed condition of dry temperate ecosystem. Pantnagar Journal of Research, 11(2): 219-224.

Kaur, S., Pembleton, L.W., Cogan, N.O.I., Savin, K.W., Leonforte, T., Paull, J., Materne, M. and Forster, J.W. (2012) Transcriptome sequencing of field pea and faba bean for discovery and validation of SSR genetic markers. BMC Genomics, 13:104.

Khichi, P., Chandan, P. M., Chauhan, J., Srinivas, J. A. and Bhagat, M. (2016). Varietal evaluation of garden pea under semi-arid onditions of Vidharba region. International Journal of Farming and allied Science, 6:20-24.

Kumar, J., Ashraf, N. and Pal, K. (2010). Variability and Character association in Garden Pea (Pisum sativum var. hortense L.). Prog. Agile. 10(1):124131

Kumar, R., Kumar, M., Dogra, R.K. and Bharat, N.K. (2015). Variability and character association studies in garden pea (Pisum sativum var. hortense L.) During winter season at mid hills of Himachal Pradesh. Legume Research. 38 (2):164-168.

Kumar, V.R. Anil, and Sharma, R.R. (2006). Character association studies in 
garden pea. Indian Journal of Horticulture, 63(2):185-187.

Lowry, O.H., Rosebrough, N.J., Farr, A.L. and Randall, R.J. (1951). Protein measurement with folin-phenol reagent. Journal of Biological Chemistry, 5:193-265.

Miller, J. D Kang, M. S. and Tai, P. Y. P. (1983). Genetic and Phenotypic Path Analyses and Heritability in Sugarcane. Crop Science, 23(4):643647.

Nawab, N.N., Subhani, G.M., Mahmood, K., Shakil, Q. and Saeed, A. (2008). Genetic Variability, Correlation and Path Analysis Studies in Garden Pea (Pisum sativum L.) Journal of Agricultural Research, 46(4):218-223.

Pal, A.K. and Singh, S. (2013). Assessment and genetic variability in garden pea (Pisum sativum L. var. hortense). International Journal of Agricultural Science, 9(1): 293-296.

Sadasivam, S. and Manickam, A. (1996). Biochemical Methods. Second Edition, Publishing New Age International (P) Limited, Publishers. pp. 190.

Sharma, M.K., Chandel, A. and Kohli, U.K. (2009). Genetic evaluation correlation coefficients and path analysis in garden pea (Pisum sativam war. hortense L.). Annals of Horticulture, 2(1):33-38.

Singh, S. K., Singh, V. P., Srivastava, S., Singh, A. K., Chaubey, B. K. and Srivastava, R. K. (2018). Estimation of correlation coefficient among yield and attributing traits of field pea (Pisum sativum L.). Legume Research-An International Journal, 41(1): 20-26.

Sood, M., Kalia, P. and Gautam, G. (2006). Heterosis, inbreeding depression and residual heterosis for pod yield, component traits and protein content in garden pea. Indian journal of Horticulture, 63(4): 460-463.

Zhu, H., Choi, H.K., Cook, D.R. and Shoemaker, R.C. (2005) Bridging model and crop legumes through comparative genomics. Plant Physiology, 137(4):1189-1196.

\section{How to cite this article:}

Umesh Thapa, Suman Nandi and Deepsil Gurung. 2020. Performance of Garden Pea (Pisum sativum var hortense L.) Genotypes and their Genetic Variation, Genetic Advance, Character Association and Path Analysis for Pod, Seed and Quality Characters. Int.J.Curr.Microbiol.App.Sci. 9(09): 3572-3586. doi: https://doi.org/10.20546/ijcmas.2020.909.442 